\title{
SPERMATOZOAN NUCLEUS LENGTH IN THREE STRAINS OF DROSOPHILA MELANOGASTER
}

\author{
R. A. BEATTY and N. S. SIDHU * \\ Agricultural Research Council Unit of Animal Genetics, Edinburgh
}

Received 26.v.66

\section{INTRODUCTION}

THIs paper describes a quantitative investigation of genetic and other effects on the length of the Drosophila spermatozoon nucleus. One objective was to detect any dependence of nucleus length on parentage in a diallel cross of three parent strains. A subsampling within the nine classes of male progeny allowed continued study of other sources of variation originally investigated by Sidhu (1963, 1964). In the absence of any direct information as to which strains would be the more profitable to investigate, strains were chosen that differed, at least, in the size of the wing cells. A further objective was to evaluate the frequency distribution of spermatozoan nucleus length within male progeny. This had two interests. First, as a prerequisite of applying analysis of variance, it was necessary to find out the extent to which the data followed a normal distribution. Second, as an important aspect of gamete genetics, was there any evidence of discontinuity in the frequency distribution, as might be caused by size differences between X-and Y-bearing spermatozoa? In addition, scaling problems were considered in interpreting the results and as a pointer for future analyses.

The experiment began with virtually no previous knowledge of patterns of genetic variation, if they existed at all, nor of the relative importance of genetic and non-genetic variation. In "operational research " of this kind, detailed biological interpretation is best deferred until a sufficient body of fact has been accumulated. Until then, the main objective is to detect repeatable statistical heterogeneities and, almost equally important, to identify sources of variation of little or no importance; in other words to display the general pattern of variation. No apology is offered for asking many questions from one body of data; a detailed approach is appropriate to exploratory work, and especially to data whose collection is more laborious than might be imagined. The paper is one of a series in which different genetic situations are explored.

\section{STOCKS, SAMPLING AND TECHNIQUE}

(i) Series 1

Strains A and B had been selected in parallel for small wing-cell size by Robertson (196r). His "Pacific" foundation stock will be

* Present address: Central Sericultural Research and Training Institute, Nazarbad; Mysore, India. 
called strain C. Flies were obtained at generation $\mathrm{I} 2$ of his selection and were increased in number by three generations of mass mating within strains. The resulting stocks, used as parents in the present work, were mated in all possible ways, giving nine classes of progeny. Progeny from parents of identical strain will be called within-strain progeny; those from parents of differing strain will be called crosses and also hetween-strain progeny. There were replicate culture bottles per class, and replicate males per bottle. All operations, including culturing on standard maize-meal molasses medium, took place in a laboratory maintained close to $19^{\circ} \mathrm{C}$. We shall sometimes refer in Series I to diallel crosses of two strains only; these are merely an abstraction from the full data of the series. The word male used alone as a noun refers always to progeny and not parents.

Culture bottles containing about i 5 parents of each sex were positioned in randomised order. After removal of the parents, progeny were collected daily from the 2nd-8th day of emergence, the male flies being segregated and kept in storage vessels until mature. (There are sufficient spermatozoa in a male between the ages of about 5-I 5 days). The order in which the males were taken, i.e. the order in which preparations were made, was randomised over all males. Permanent aceto-orcein preparations were made under machine pressure, and the longer axis of the spermatozoan nucleus was measured from drawings made under optical projection at a linear magnification of $\times 6950$. The detailed procedures are given by Sidhu (1964). The drawings were measured in " projection units " of $\mathrm{I} / 32$ ", corresponding to units of $0 \cdot I_{1} 2 \mu$ in the nucleus itself, a unit being about one-tenth of the within-male standard deviation. The high magnification, and the use of a unit of measurement smaller than the resolving power of the microscope, were intended to minimise observational error. Calculations are presented on a basis of microns $(\mu)$.

\section{(ii) Series II and III}

Series II was a repetition of Series I, with the same subsampling, but carried out after three more generations of relaxed selection and with only strains B and C used as parents. Series III data were obtained by re-measuring some of the Series II slides.

\section{STATISTICAL METHODS, SYMBOLS}

Two main attributes of the spermatozoan phenotype were of interest: the nucleus length and the within-male variance in nucleus length. Analysis of the variance of variance was conducted after transforming each variance into its logarithm, a theoretical variance of log variances being available for such analyses (Rao, I952). In Series III two further attributes of spermatozoa were also analysed; these were the coefficient of skewness in the within-male distribution of nucleus length $\left(g_{1}\right)$, and the coefficient of kurtosis $\left(g_{2}\right)$ in the same distribution. 
Sources of variation affecting these four attributes were examined in some detail, the present work being exploratory in nature. Logarithms to the base ro were used, and log transformation of original data, i.e. of each measurement of a nucleus, is to be distinguished from the log transformation of variances applied before analysis of the variance of variance.

\section{(i) Sources of variation in a diallel cross}

The three strains are regarded as a fixed group not representing any meaningful larger population of strains. If parental strains are mated in all possible ways, including reciprocals, the variance of the means of the progeny classes can be partitioned into the following items by the method of Hayman (r954) (see also Wearden, r 964):-

$a$ (parental strains), containing the genic variation common to both sexes; $b$ (genetic interaction), being the variation in reciprocal sums not due to $a ; c$ (average maternal effects confounded with the analogous "paternal effects"), being differences between the effects of each strain used as a male parent and as a female parent; $d$ (reciprocal effect), being variation in reciprocal differences not due to $c$; and an estimate of error, called $\mathrm{M}(\mathrm{G})$ in the present work. Variance components in the diallel can be isolated-or more strictly, "variances of fixed effects ", in Wearden's sense. Since in every analysis $d$ was found to be non-significant when tested against $\mathrm{M}(\mathrm{C})$ and $c$ was also non-significant when tested against $d$ or $\mathbf{M}(\mathbf{C})$, it was assumed that reciprocal and maternal (or "paternal") effects had variance components of zero, and the analyses are presented with $a, b, c$ and $d$ each tested against $\mathbf{M}(\mathbf{C})$. In one analysis $b$ is further partitioned into $b_{1}$ (mean of all crosses $v$. mean of all within-strain progeny, corresponding to Hayman's " mean dominance") and $b_{2}$ (remaining genetic interaction). In addition, a non-orthogonal test can be made for testing differences between means of within-strain progeny classes.

If the spermatozoon is regarded merely as a piece of the diploid male soma, as we shall assume for purposes of exposition, the normal methods of diallel analysis are applicable. There is not much evidence in the literature that the haploid gene complement affects the spermatozoan phenotype. Even if there were a haploid effect, it would still seem reasonable to apply the methods in pursuit of our main objective, the detection of patterns of variation in the data, though there would be new genetic parameters that we are not ready to visualise yet.

\section{(ii) Sources of variation in the subsampling}

The subsampling provides an error term for testing differences between classes of progeny, but otherwise may be considered separately. Symbols are: spermatozoa within males, $\mathrm{S}(\mathbf{M})$; males within classes of progeny, $\mathbf{M}(\mathrm{C})$. The latter can be partitioned into males within culture bottles, $\mathbf{M}(\mathbf{B})$, and culture bottles within classes of progeny, $\mathrm{B}(\mathrm{C})$. 


\section{(iii) Tests of significance}

Special reliance is to be placed on tests planned in advance; these were for $a, b, c, \mathbf{M}(\mathbf{C}), \mathrm{B}(\mathrm{C}), \mathbf{M}(\mathrm{B})$ and for differences between withinstrain progeny means. The error terms are named in the analyses but the reasons for their choice, depending on the sampling structure and on judgments about the reality or otherwise of various components of variance, will not always be given. Significance levels are marked as follows:-

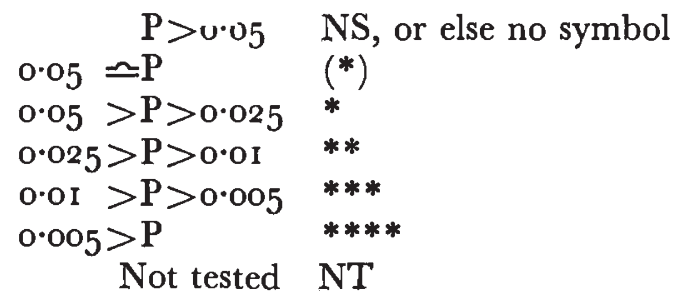

\section{RESULTS : DIALLEL CROSS OF STRAINS A, B AND C (SERIES I DATA)}

The sampling was: three strains, giving nine classes of progeny (table I); three culture bottles per class; four male offspring per bottle; ten spermatozoa (on one microscope slide) per male; total I 080 spermatozoa whose nuclei were measured.

TABLE I

Mean spermatozoan nucleus length in nine classes of progeny from the diallel cross of strains $A, B$, and $C$; Series I data. S.E. is derived from the $M(C)$ mean square in table 2

\begin{tabular}{|c|c|c|c|c|}
\hline \multirow[b]{3}{*}{$\delta$ parents } & \multirow[b]{3}{*}{$\begin{array}{l}\mathrm{A} \\
\mathrm{B} \\
\mathrm{C}\end{array}$} & \multicolumn{3}{|c|}{ q parents } \\
\hline & & A & B & $\overline{\mathrm{C}}$ \\
\hline & & $\begin{array}{l}9 \cdot 3 \text { O I } \\
9 \cdot 363 \\
9 \cdot 25^{8}\end{array}$ & $\begin{array}{l}9 \cdot 549 \\
9 \cdot 683 \\
8 \cdot 853\end{array}$ & $\begin{array}{l}9 \cdot 227 \\
8 \cdot 716 \\
9 \cdot 655\end{array}$ \\
\hline \multicolumn{2}{|c|}{ S.E. (99 d.f.) per class mean } & \multicolumn{3}{|c|}{$\pm \cdot 273$} \\
\hline \multicolumn{2}{|c|}{ Grand mean } & \multicolumn{3}{|c|}{$9 \cdot 290$} \\
\hline
\end{tabular}

Differences among the nine means of progeny classes of the diallel cross are analysed in the upper part of table 2, where it will be seen that no significance is attached to the items for parental strains $(a)$, maternal effects $(c)$ or reciprocal effects $(d)$, all of which were taken to have variance components of zero. The significant genetic interaction $(b)$ has a variance component of 0.07 and when partitioned yields two significant mean squares: $b_{1}$, testing mean difference between all crosses and all within-strain progeny; and $b_{2}$, testing remaining genetic 
TABLE 2

Analysis of variance of mean spermatozoan nucleus length in the diallel cross of strains $A, B$, and $C_{2}$ Series I data. Items to the right of a large bracket are a partioning of the item to its left. For significance levels, see p. 68

\begin{tabular}{|c|c|c|c|c|}
\hline \multicolumn{4}{|c|}{ Source of Variation } & \multirow[b]{2}{*}{$\begin{array}{c}\text { Mean } \\
\text { square }\end{array}$} \\
\hline Symbol & d.f. & Name & $\begin{array}{c}\text { Tested } \\
\text { to: }\end{array}$ & \\
\hline \multicolumn{5}{|c|}{ (Nine classes of progeny in diallel) } \\
\hline${ }_{c}^{a} \begin{array}{l}a \\
b_{1} \\
b_{2}\end{array}$ & ${ }_{2}^{3}\left\{\begin{array}{l}1 \\
2\end{array}\right.$ & $\begin{array}{l}\text { Parental strains } \\
\text { Genetic interaction } \\
\text { Crosses } v \text {. within-strain } \\
\text { Remaining genetic interaction } \\
\text { Maternal effects } \\
\text { Reciprocal effects }\end{array}$ & $\begin{array}{l}M(C) \\
M(C) \\
M(C) \\
M(C) \\
M(C) \\
M(C)\end{array}$ & $\begin{array}{l}2 \cdot 20 \\
32 \cdot 88^{* *} \\
35 \cdot 69^{*} \\
31 \cdot 48^{*} \\
1 \cdot 56 \\
0 \cdot 13\end{array}$ \\
\hline \multicolumn{5}{|c|}{ (Subsampling) } \\
\hline $\begin{array}{l}M(C)\left\{\begin{array}{l}B(C) \\
M(B)\end{array}\right. \\
S(M)\end{array}$ & $99\left\{\begin{array}{l}\mathrm{I} 8 \\
8 \mathrm{I}\end{array}\right.$ & $\begin{array}{l}\text { Males in progeny classes } \\
\text { Bottles in progeny classes } \\
\text { Males in bottles } \\
\text { Spermatozoa in males }\end{array}$ & $\begin{array}{l}\mathrm{S}(\mathbf{M}) \\
\mathbf{M}(\mathbf{B}) \\
\mathbf{S}(\mathbf{M}) \\
-\end{array}$ & $\begin{array}{l}8 \cdot 94^{* * * *} \\
7 \cdot 81 \\
9 \cdot 19^{* * * *} \\
1 \cdot 4^{8} 7\end{array}$ \\
\hline
\end{tabular}

interaction, visualisable as heterogeneity between the various $b$ calculable for particular pairs of parents. The interaction is illustrated by actual figures in table 3 , where it is shown that the difference (crosses) - (between-strain progeny) is highly significant for the diallel of strains

TABLE 3

The difference in mean spermatozoan nucleus length between crosses (=between-strain progeny) and within-strain progeny, for the diallel cross of strains $A, B$, and $C$ and for diallels of parental strains considered two at a time; Series I data. S.E. is derived from the $M(C)$ mean square in table 2. For significance levels see $p .68$

\begin{tabular}{|c|c|c|c|c|}
\hline & \multicolumn{4}{|c|}{ Parental strains in diallel } \\
\hline $\begin{array}{l}\text { Mean of crosses } \\
\text { Mean of within-strain progeny }\end{array}$ & $\begin{array}{l}9 \cdot 16 \mathrm{I} \\
9 \cdot 547\end{array}$ & $\begin{array}{l}9 \cdot 45^{6} \\
9 \cdot 49^{2}\end{array}$ & $\begin{array}{l}9 \cdot 242 \\
9 \cdot 478\end{array}$ & $\begin{array}{l}8 \cdot 785 \\
9 \cdot 669\end{array}$ \\
\hline Difference & $-0 \cdot 3^{86 *}$ & -0.036 & -0.236 & $-0.885^{* * * *}$ \\
\hline S.E. of difference (99 d.f.) & $\pm 0 \cdot 193$ & & \pm 0.273 & \\
\hline
\end{tabular}

$\mathrm{B}$ and $\mathrm{C}\left(-0 \cdot 885^{* * * *} \pm 0.273 \mu\right)$, while differences for the diallel of $\mathrm{A}$ and $\mathrm{C}$ only, or $\mathrm{A}$ and $\mathrm{B}$ only, though also negative, are non-significant. $b_{1}$ is not therefore an item of much interest, since it is largely a E 2 
reflection of the interaction of $\mathrm{B}$ and $\mathrm{C}$ alone. Analyses of variance (not shown) of the log within-male variance yielded no significant differences between progeny classes.

In the subsampling in the lower part of table 2, there are highly significant differences in mean spermatozoan nucleus length between males within classes of progeny. When this source is partitioned, there result highly significant differences between males within culture bottles (variance component $0 \cdot 77$ ), no significant differences between culture bottles within classes of progeny (variance component zero), and there is high variability of spermatozoa within males (variance component I.49). Analyses (not shown) of the log within-male variance showed a pattern of variation similar to that of mean nucleus length.

The mean nucleus length per progeny class and the square root of the pooled within-male variance per progeny class were highly correlated $\left(r=+0 \cdot 85^{* * * *}\right)$ but in log-transformed original data this became $+0.4 \mathrm{I}$ (not significant). Otherwise, analyses of untransformed and transformed original data gave analogous results. The pooled withinmale variance (I 3 per cent. of the arithmetic mean in untransformed data) was virtually the same in transformed data (I 4 per cent. of the geometric mean).

\section{RESULTS: DIALLEL CROSS OF STRAINS B AND C ONLY: ADDITIONAL OBSERVATIONS (SERIES II) AND JOINT ANALYSIS WITH PART OF SERIES I}

Series I yielded our first real evidence of a genetic effect on the spermatozoan phenotype, the progeny from crosses of strains $B$ and $\mathrm{C}$ having shorter spermatozoan nuclei than did the progeny from within-strain matings of the same strains. A check was carried out as follows. A wholly new experiment (Series II) was carried out exactly as in Series I, but omitting strain A, and after three unavoidable additional generations of relaxed selection. Data for a diallel cross between strains B and C only were taken from the record sheets of Series I. The two symmetrical sets of data were then analysed simultaneously. The means of classes of progeny in Series I are at the top of table 4, the means from Series II in the middle, and the average of the two series at the bottom.

Consistency between the two series was a vital first consideration. Preliminary tests showed no significant series difference between the mean squares for $\mathrm{M}(\mathrm{B})$; they were therefore pooled to yield the joint mean square in line 8 of table 5. Pooling over series was justified and effected in the same way for $\mathbf{M}(\mathbf{C})$ and $\mathbf{B}(\mathrm{C})$. The overall means of the two series did not differ significantly (line 4). Three series interactions were not significantly heterogeneous nor individually significant; they were also not significant when pooled (line 5). These 
detailed tests gave good grounds for assuming that the two series were consistent and could validly be combined.

TABLE 4

Mean spermatozoan nucleus length per class of progeny from parental strains $B$ and $C$ : data from Series $I$ and II shown separately and combined. S.E. is derived from line 6 of table 5

\begin{tabular}{|c|c|c|c|c|}
\hline & & & \multicolumn{2}{|c|}{$\begin{array}{c}\stackrel{+}{ } \\
\text { parents }\end{array}$} \\
\hline & & & B & $\mathrm{C}$ \\
\hline $\begin{array}{l}\text { Series I data from table I } \\
\text { Series II data }\end{array}$ & $\begin{array}{l}\delta \text { parents } \\
\text { ô parents }\end{array}$ & $\begin{array}{l}\text { B } \\
\text { C } \\
\text { B } \\
\text { C }\end{array}$ & $\begin{array}{l}9 \cdot 683 \\
8 \cdot 853 \\
9 \cdot 046 \\
8 \cdot 946\end{array}$ & $\begin{array}{l}8 \cdot 716 \\
9 \cdot 655 \\
8 \cdot 799 \\
9 \cdot 235\end{array}$ \\
\hline Series I and II combined & $\begin{array}{l}\text { đ parents } \\
\text { S.E. per mean, } 88 \text { d.f. } \\
\text { Grand mean }\end{array}$ & $\begin{array}{l}\text { B } \\
\text { C }\end{array}$ & $\begin{array}{r}9 \cdot 365 \\
8 \cdot 900 \\
\pm\end{array}$ & $\begin{array}{l}8 \cdot 757 \\
9 \cdot 445 \\
5 \\
7\end{array}$ \\
\hline
\end{tabular}

The final analysis of untransformed joint data is set out in table 5 . A highly significant genetic interaction emerged. (Significance sur-

\section{TABLE 5}

Mean spermatozoan nucleus length in a joint analysis of Series $I$ and II data for the diallel cross of strains $B$ and $C$ only. For significance levels see p. 68. Items to the right of a large bracket are a partitioning of the item to its left

\begin{tabular}{|c|c|c|c|c|c|}
\hline \multirow{2}{*}{ Line } & \multicolumn{4}{|c|}{ Source of variation } & \multirow{2}{*}{ Mean squares } \\
\hline & Symbol & d.f. & Name & $\begin{array}{c}\text { Tested } \\
\text { to: }\end{array}$ & \\
\hline $\begin{array}{l}\text { I } \\
2 \\
3 \\
4 \\
5 \\
6 \\
7 \\
7 \\
9\end{array}$ & $\begin{array}{l}\text { C }\left\{\begin{array}{l}a+c \\
b\end{array}\right. \\
M(C)\left\{\begin{array}{l} \\
B(C) \\
M(B)\end{array}\right.\end{array}$ & $\begin{array}{l}\left\{\begin{array}{l}2 \\
I\end{array}\right. \\
3 \\
88\left\{\begin{array}{l}I \\
16 \\
72\end{array}\right. \\
864\end{array}$ & $\begin{array}{l}\text { Classes of progeny } \\
\text { Pooled parental strain } \\
\text { and maternal effects } \\
\text { Genetic interaction } \\
\text { Series I } v \text {. II } \\
\text { Pooled series interac- } \\
\text { tions } \\
\text { Males in progeny } \\
\text { classes } \\
\text { Bottles in classes } \\
\text { Males in bottles } \\
\text { Spermatozoa in males }\end{array}$ & $\begin{array}{l}M(G) \\
M(G) \\
M(G) \\
M(G) \\
M(G) \\
- \\
M(B) \\
-\end{array}$ & $\begin{array}{l}27 \cdot 64^{*}\left\{\begin{array}{l}1 \cdot 60 \\
79 \cdot 70^{* * * *}\end{array}\right. \\
\begin{array}{l}8.07 \\
9 \cdot 65\end{array} \\
\text { Not computed }\left\{\begin{array}{l}1.7 \mathrm{I} \\
8 \cdot 60^{N T}\end{array}\right.\end{array}$ \\
\hline
\end{tabular}

vived even a more stringent test employing an alternative error term with only 4 d.f., obtained by pooling lines 4 and 5 ). Since this was a planned comparison, the validity of the test of significance does not require that the overall differences $(C)$ between the four classes of 
progeny should necessarily be significant, but it was reassuring to find that $\mathrm{C}$ was, in fact, significant. The parental strain effect and maternal effects were each non-significant and were pooled to give the mean square in line 2. An additional test showed no significant difference between the two classes of within-strain progeny. These findings reinforce the original conclusions from Series $I$ alone. $M(C)$ and $M(B)$ are not tested in Series II, and $\mathbf{S}(\mathbf{M})$ is not computed. The general pattern of variation was virtually the same in parallel analyses of logtransformed original data.

The very strong evidence of genetic interaction is easily visualised in the three $2 \times 2$ tables of table 4 , in each of which each cross has shorter nuclei than in each class of within-strain progeny. There seems no room for doubt about the smaller size of nuclei in crosses. The final estimate from the combined results of the two series is that the mean nucleus length is $8.829 \mu$ over all crosses and $9.405 \mu$ over all withinstrain progeny, the difference being $0.576^{* * * *} \pm 0.195 \mu$ (88 d.f.). This supersedes the earlier estimates on the right of table 3 , which played a part in developing the argument and the experiments. Negative results are, that the two series agree at all levels in showing no real evidence of a parental strain effect or of maternal effects when strains $\mathrm{B}$ and $\mathbf{C}$ are crossed in all possible ways, nor of any differences between culture bottles or between the grand means of the series.

\section{RESULTS: THE FREQUENCY DISTRIBUTION OF SPERMA- TOZOAN NUCLEUS LENGTH WITHIN MALES IN THE DIALLEL CROSS OF STRAINS B AND $C$ ONLY: SERIES III DATA}

Larger numbers of spermatozoa per male were measured than in Series I and II, in order to obtain adequate estimates of the higher moments of the frequency distribution. The numbers of males sampled had to be reduced in order to keep the work within practical bounds. From the preparations already made in Series II from progeny of a diallel cross of strains $\mathrm{B}$ and $\mathrm{C}$, slides containing inadequate numbers of spermatozoa were rejected. A random selection of the remaining slides gave a balanced sampling: two culture bottles in each of the four classes of progeny; two males per culture bottle; 50 spermatozoa (on one microscope slide) per male; total, 800 spermatozoa whose nuclear lengths were measured. The various techniques and precautions of Series II were followed. The original data are recorded in more detail by Sidhu (1963).

The statistical basis of nearly all that follows is that of Fisher (1930). The procedures are summarised with particular reference to spermatozoa by Beatty $(\operatorname{Ig} 6 \mathrm{I} a)$.

The following statistics of the spermatozoan nucleus length were calculated for each of the 16 male progeny: the mean; the within-male variance; the coefficient of skewness, $g_{1}$ (positive for an excess of items 
smaller than the mean, i.e. "long tail to the right"; negative for a deficiency of such items); the coefficient of kurtosis, $g_{2}$ (negative for a distribution curve broad at the shoulders and depleted at the tails, a special case being a bimodal curve; positive for narrow shoulders and extended tails). In the "normal distribution", $g_{1}=g_{2}=0$. Class means are summarised in table 6 .

\section{TABLE 6}

Characteristics of the frequency distribution of spermatozoan nucleus length in the four classes of progeny of a diallel cross of strains $B$ and $C$ only, Series III data. The significance levels (see p. 68) are for the departure of skewness and kurtosis from zero. S.E. is derived from line 4 of table 7

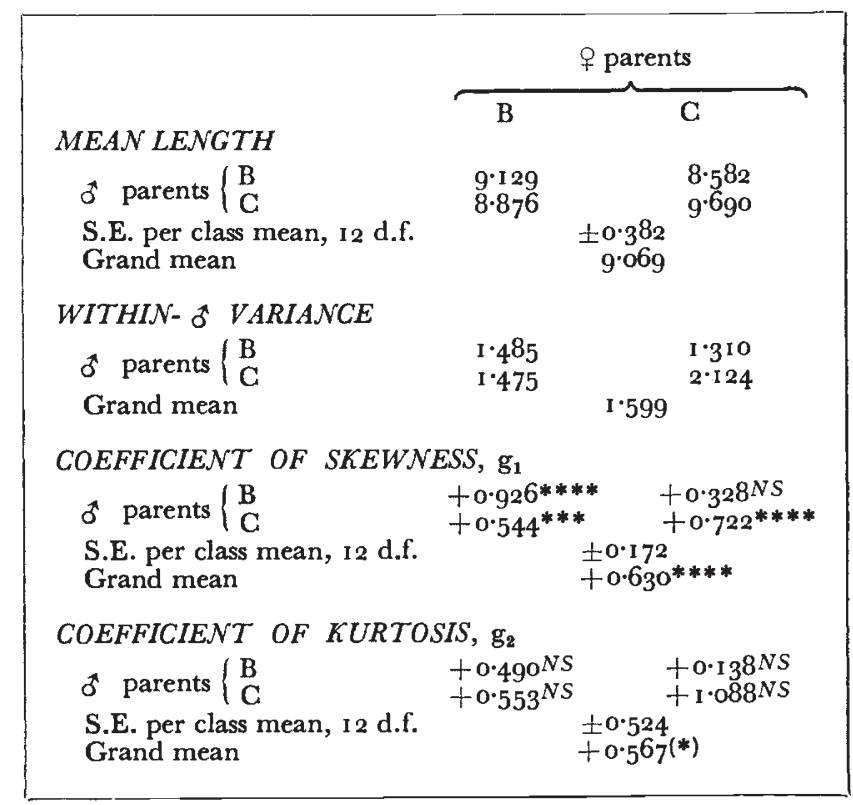

These statistics can be regarded as four attributes of spermatozoa, each subject to the influence of sources of variation described on p. 67 . Each attribute was subjected to an analysis of variance, of which three are shown in table 7. The mean, $g_{1}$ and $g_{2}$ were analysed as such, while each within-male variance was first transformed into its logarithm. The theoretical variances of $g_{1}$ and $g_{2}$ were calculated from Fisher's formulæ, while that of the $\log$ within-male variance was as on p. 66. A mean square on I d.f. (the "correction term") was entered for testing the significance of the departures of the grand means of $g_{1}$ and $g_{2}$ from zero. The pooled variation in line 2 was partitioned into $a$ and $c$, but the resulting mean squares were nonsignificant.

\section{(i) Results of analysis}

Mean spermatozoan nucleus length (table 6) shows the same trends as in Series I and II, the nucleus being shorter in crosses than in within- 
R. A. BEATTY AND N. S. SIDHU

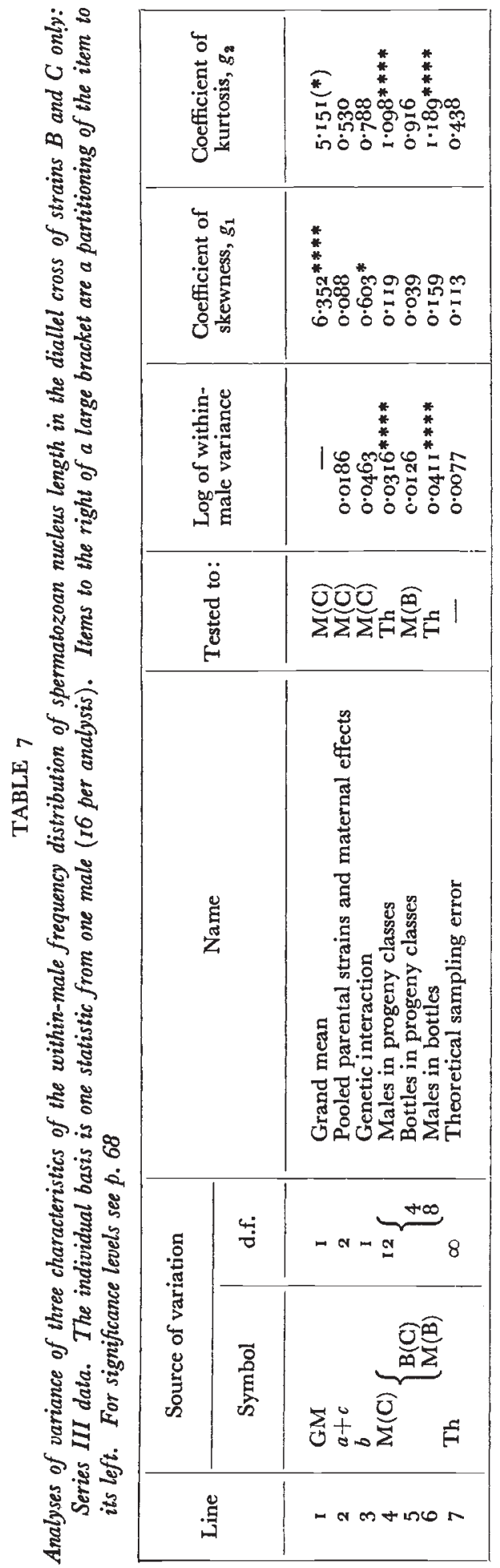


strain progeny. Analyses, not presented here, showed that the trend was not significant, but this need not be regarded as a discrepancy, because the S.E. per class mean is higher than in Series I and II and is based on only I 2 d.f. This lesser precision is because Series III was designed to produce estimates of the higher moments and became less suitable for analysis of the first moment. In the subsampling, the same sources of variation were significant as in Series I and components of variance were of the same order.

The (log) within-male variance in nucleus length is analysed in table 7. There are highly significant differences between males within progeny classes. When this source is partitioned, males within bottles differ at a high level of significance, but there are no significant differences between culture bottles within progeny classes. There is no significant variation in the upper part of the table (parental strains, maternal effects, interaction). As in Series I, log-transformation of the original data was found to reduce the correlation between mean nucleus length and the square root of the pooled within-male variance per progeny class.

The coefficient of skewness; $g_{1}$, shows a marked positive tendency ("long tail to the right") in table 6, less extreme in crosses than in within-strain progeny. This is reflected in significant form in lines I and 3 of the analysis in table 7 . Skewness is necessarily affected by the scale of measurement. The grand average in table 6 is $+0.630 * * * *$, and was found to be $+0 \cdot 308 * * * *$ in a parallel analysis of log-transformed original data, while a log-log transformation of original data was found to give a negative $g_{1}$. There is less effect of scale on the significant differences in skewness for the mean of crosses $v$. the mean of within-strain progeny, which is $-0.388^{*}$ from table 6 and was found to be $-0.402 * * *$ from log-transformed original data. This supports the hypothesis that there is an essential difference in skewness, not dependent on the scaling. In the subsampling there is no significant effect of any kind.

The grand mean of the coefficient of kurtosis, $g_{2}$ yields ultimately identical tests of significance in tables 6 and 7 verging on the formal level of significance of $\mathrm{P}<0 \cdot 05$. The validity of this unplanned comparison is, however, doubtful. As in the analysis of $(\log )$ within-male variance, a highly significant item for males within classes of progeny can be partitioned into a highly significant item for males within bottles and a non-significant item for bottles within progeny classes. (Parallel analyses of log-transformed original data showed a dramatic change; no source of variation was significant, and kurtosis could be considered as uniformly close to zero at all levels of the analysis).

\section{(ii) Do $X$ - and $Y$-bearing spermatozoa differ in nucleus length?}

There was no clear evidence of dimegaly (two size classes) when frequency distribution curves were plotted for each of the 16 male 
progeny, nor in the average within-male distributions shown in fig. I. The following special analysis was therefore applied in a search for possible dimegaly obscured by sampling error. If a given frequency

NUCLEUS LENGTH IN $\mu$

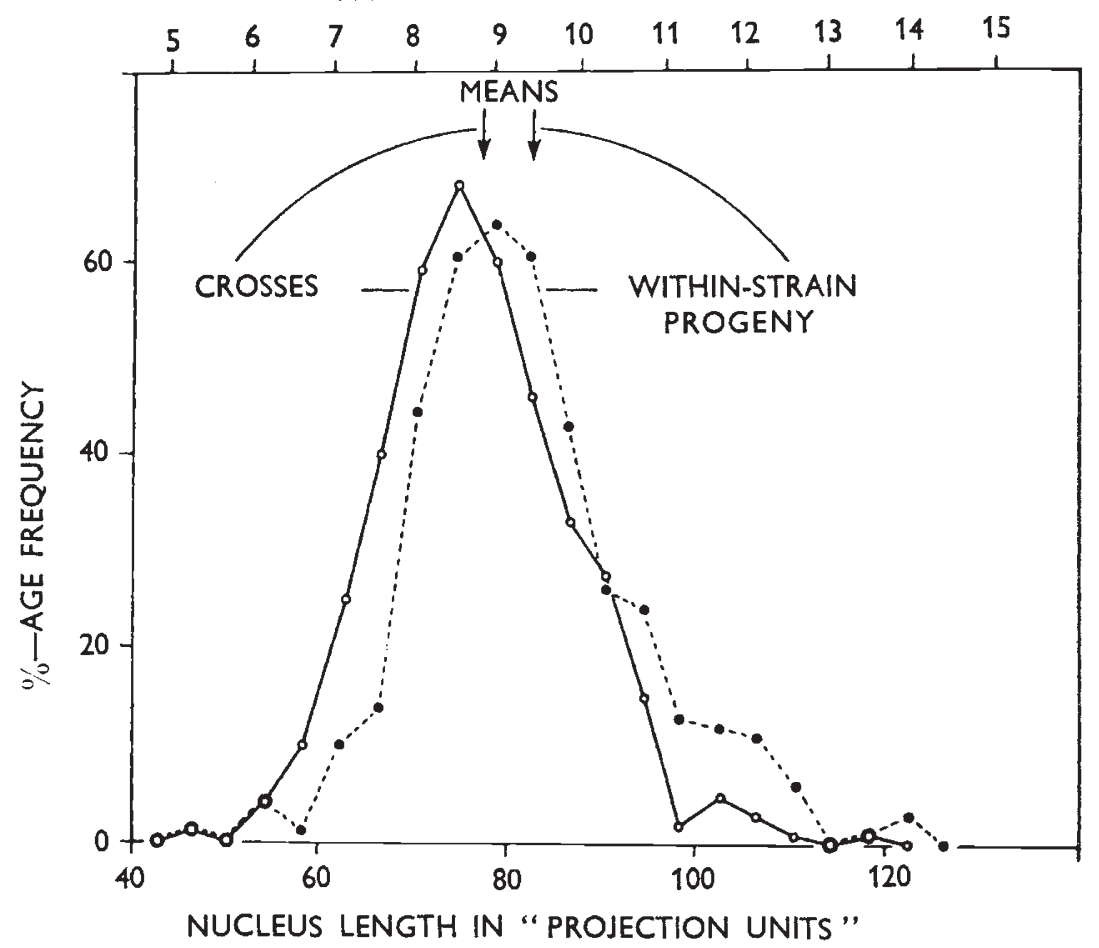

FIG. I.-Illustration of the within-male frequency distribution of spermatozoan nucleus length of crosses (hollow circles and full lines) compared with within-strain progeny (solid circles and dashed lines) for the diallel of strains B and C. Individual frequency tables for crosses in Series III were expressed as deviations around zero, pooled, fitted to the mean for crosses in the joint data of Series I and II $(8.829 \mu, \mathrm{p} .72)$ and finally regrouped in coarser class intervals. Within-strain data were fitted correspondingly to the mean of $9.405 \mu$. These means of p. 72 were preferred as being better determined than the actual means of Series III in table 6 . Class centres are plotted at values $42.5,46.5 \ldots$. 126.5 of the scale in "projection units", and a scale in $\mu$ is also provided.

distribution is in reality a compound distribution resulting from the summation of two equal and normally distributed underlying distributions whose means differ by a value $\mathrm{D}$, then $\mathrm{D}=\sqrt[4]{-8 k_{4}}$, where $k_{4}$ is the fourth cumulant (unpublished formula of E. G. R. Reeve, quoted in Beatty, $196 \mathrm{r} a)$. This can be written in the form $\mathrm{D}=\mathrm{SD} \times \sqrt[4]{-8 g_{2}}$, where $\mathrm{SD}$ is the true population standard deviation and $g_{2}$ the coefficient of kurtosis, both of the compound distribution. When SD is substituted by a well determined estimate, as in the present work, approximate upper and lower confidence limits for D can be derived after setting confidence limits to $g_{2}$ alone. D is best estimated as zero if, as sometimes happens, the formula when applied to data leads to a figure depending on the fourth root of a negative quantity. The for- 
mula is equally appropriate to the special case of a symmetrically bimodal curve.

Data for the analysis, from table 6, were: $g_{2}=$ grand mean of $g_{2}$; $\mathrm{SE} g_{2}=$ (SE per class mean of $\left.g_{2}\right) / 2 ; n=\mathrm{I} 2$ d.f.; $\mathrm{SD}=$ square root of the grand mean of the within-male variance. Confidence limits to $g_{2}$ were at the $0.05 \mathrm{P}$ level $\left(t_{[n]}=2 \cdot 18\right)$. The grand mean of $g_{2}$ being positive, the estimate of $\mathrm{D}$ and of its lower confidence limit are both taken to be zero. The upper confidence limit of D works out as $0.52 \mu$, as if the observed grand mean of spermatozoan nucleus length of $9.07 \mu$ (table 6) could be the average of two underlying means of $8.8 \mathrm{I} \mu$ and $9 \cdot 33 \mu$. The assumptions behind the formula are met to the following extent. We can rely on Mendelian expectation for the presence of virtually equal numbers of $\mathrm{X}$ - and $\mathrm{Y}$-bearing spermatozoa. It remains an assumption that each underlying mean is normally distributed and, in fact, the observed compound distribution is positively skewed, but the calculation is thought to be tolerant of skewness. SD is obtained from observations; however, we know from several experiments that it cannot be far from the true value, and error in the determination of SD should contribute only a negligible bias to the calculations.

In short, there is no evidence of two classes of spermatozoa differing in mean nucleus length, and hence no evidence of dimegaly attributable to X-and Y-bearing spermatozoa or to any other cause. Sampling error was such that dimegaly might well have escaped notice if the one underlying mean exceeded the other by less than some 6 per cent. (or io per cent. from a parallel calculation based on log-transformed original data).

\section{CONCLUSIONS AND DISCUSSION}

\section{(i) The parent strains}

Strains A and B were received after independent and successful selection for small wing-cell size, small body size (thorax length) being a correlated response. Strain $\mathrm{C}$ was the mass-mated control. The strains are represented in the diallel crosses by the three classes of within-strain progeny, between which there are no demonstrable differences in mean spermatozoan nucleus length. There is, therefore, no evidence that either the selection for wing-cell size or the resulting diminution in body size brought about any correlated response in spermatozoan nucleus length. The within-strain progeny of $\mathrm{A}$ and $\mathrm{B}$ are not precisely equivalent to the strains originally received because several generations of relaxed selection had intervened. On the other hand, A and B as received were necessarily somewhat inbred as a result of selection, and one would not expect the relaxed selection to have completely destroyed any correlated response. In mice, independence of body size and spermatozoan size have been shown in strains selected for large body size (Beatty, 1963 ), and Beatty ( $196 \mathrm{I} b$ ) 
has stressed that various sources of variation affect body size and spermatozoan dimensions in different ways.

Though selected in parallel, strains A and B showed a different genetic interaction with strain $\mathrm{C}$. Hence, one or some of the genes governing nucleus length are not the same in $\mathrm{A}$ and $\mathrm{B}$, possibly because of random drift.

\section{(ii) Characteristics of the frequency distribution of spermatozoan nucleus length}

Essentially, the "Results" sections have been about the influence of sources of variation on four characteristics of the frequency distribution of spermatozoan nucleus length. There are advantages in first considering the four characteristics (in the present section), and then the sources of variation, so that the same facts are reviewed in two different perspectives.

Mean spermatozoan nucleus length is subject to highly significant variation between males within culture bottles. The only other significant source is genetic interaction, between-strain progeny having smaller nuclei than within-strain progeny in the diallel cross of strains $B$ and C. No significant interaction occurs in the diallel of $\mathbf{A}$ and $\mathbf{C}$ only, or of A and B only. All these conclusions are the same in untransformed and log-transformed data.

The within-male variance in nucleus length, both in untransformed and log-transformed data, varies significantly between males within culture bottles, and no other source of variation is significant. The within-male variance (as its square root) per class of progeny and the mean nucleus length are strongly and positively correlated in untransformed data, while in transformed data the correlation is diminished and is non-significant. Log transformation does not diminish the relative magnitude of the grand mean of the within-male variance, which is represented by a coefficient of variation of 13 per cent. in untransformed data and I4 per cent in transformed data. Sidhu (I964) has given reasons for considering most of this high variance to be a real attribute of spermatozoa which cannot be reduced in magnitude by transformation or by changing the techniques of preparation and measurement. This means that all analyses of mean nucleus length in Drosophila spermatozoa contain in the expectation of each mean square an unavoidably large error component whose magnitude can be diminished only by measuring larger numbers of spermatozoa. A point of more biological interest is that part of this high within-male variance may, conceivably, express effects of genetic factors in the haploid genotype of the developing spermatozoa themselves.

The coefficient of skewness, $g_{1}$, studied only in the diallel cross of strains B and C, has a grand mean that is significantly positive ("long tail to the right"), especially in untransformed data. The only other significant source of variation is genetic interaction, $g_{1}$ in within-strain progeny being significantly more positive than in between-strain pro- 
geny, both in untransformed and transformed data. The coefficient of kurtosis, $g_{2}$, studied only in the diallel cross of strains B and C, has a grand mean that differs only doubtfully from zero in both untransformed and transformed data. A heterogeneity in $g_{2}$ between males within culture bottles is evident in untransformed data but vanishes in transformed data. No other sources of variation in $g_{2}$ are certainly significant.

\section{(iii) Factors affecting the frequency distribution of spermatozoan nucleus length}

Genetic effects on the progeny class means in the diallel cross of strains $B$ and $\mathbf{C}$ are represented solely by interaction affecting both the mean nucleus length and the coefficient of skewness: there is no sign of a significant effect of parental strains or of maternal effects on the mean nucleus length, the within-male variance, $g_{1}$ or $g_{2}$. These conclusions were the same for untransformed and transformed data. The same remarks apply to a full diallel cross of all three strains (except that $g_{1}$ and $g_{2}$ were not studied) but with the additional information that reciprocal effects were non-significant. Our main genetic finding, illustrated in fig. I, is that within-strain progeny in the diallel cross of strains B and C have spermatozoan nuclei that are longer and distributed in a more positively skew fashion than those of between-strain progeny.

Subsample effects. In untransformed and transformed data, the mean nucleus length and the within-male variance (but not the skewness) each differ significantly between males within culture bottles. There are also differences in kurtosis between males within culture bottles in untransformed data, but these vanish in transformed data. There are no differences between culture bottles for any aspect of the frequency distribution. This latter finding has two implications. First, spermatozoan nucleus size must be independent of various environmental imponderables that certainly distinguished one culture bottle from another and would be expected to affect other characteristics, such as body size. Second, replication of culture bottles is probably unnecessary in studying nucleus length, though a minimal replication might be retained as a safeguard. Finally, we must stress again that only one microscope preparation was made from each male, so that the observed male-in-bottle effect is compounded of two possible effects: a true male-in-bottle effect, and a preparation-in-male effect. It will be shown in another paper that the preparation-in-male effect is large and probably reflects variability in technique.

In Series III, a special analysis showed no significant evidence of the presence of two main classes of nucleus length within each male, such as might be attributable to a difference between $\mathrm{X}$ - and Y-bearing spermatozoa, or to some other cause. However, sampling error could have obscured any real difference between size classes if the one mean exceeded the other by less than some 6-ro per cent. 


\section{(iv) Scaling}

In untransformed data, the within-male distribution of nucleus length is positively skew, the within-male variance is positively correlated with the mean and is heterogeneous between males within bottles, there is a suspicion that the average kurtosis is positive, and there are highly significant differences in kurtosis between males within culture bottles. In log-transformed data, all these tendencies are diminished, the significant heterogeneity in kurtosis vanishing altogether. These findings indicate that the assumptions underlying the application of the technique of analysis of variance are more closely obeyed if the original data are first transformed into logarithms. However, analysis of variance is tolerant of moderate departures from assumption and, in fact, the recognition of a source as significant or non-significant was usually unaffected by transformation. Transformation had virtually no effect on the percentage components of variance in the subsampling. The data did not seem to call for exploration of any kind of transformation other than the log transformation.

\section{(v) Repeatability of results}

There is little previous literature about the detailed pattern of variation in the phenotype of Drosophila spermatozoa. In addition, sampling designs have to be arranged carefully to accommodate the existence of errors in nucleus measurement attributable to observation, technique and the limits of optical resolution. At this stage of the work, therefore, we pay special attention to confirmation of results by repetition. This gives an additional reason for analysing each set of results in detail. In the present work, the interaction trends were the same in Series I and the independent Series II (as well as in Series III, semi-independent of Series II). Further, at all points of repetition, there is agreement between the present work and that of Sidhu (Ig64); mean spermatozoan nucleus length differs between males within culture bottles, but not between culture bottles within classes of progeny; variance components in the subsampling are of comparable magnitude; there is evidence of heterogeneity of variance between males within culture bottles; a logarithmic transformation of the original data diminishes a correlation between the mean and the within-male variance.

\section{(vi) The pattern of variation in the phenotype of Drosophila spermatozoa}

In quantitative studies of, say, body size, there is no need in any species to prove the obvious fact that body size varies in response to various factors. In these initial studies of Drosophila spermatozoa there was little background knowledge except for an analogy with results from mammalian spermatozoa, and nothing could be taken for granted. This background is being built up as the work progresses. We now know that spermatozoan nucleus size in Drosophila does vary in accordance with various sources of variation; this is now obvious, but was not 
so before. The general pattern of response to sources of variation is not the same in spermatozoan nucleus length as in body size. In mice and rabbits (Beatty, 1961 $b$ ) spermatozoan dimensions are remarkably independent of environmental effects, and so far this appears to hold true for the nucleus length in Drosophila spermatozoa. A detailed comparison with mammalian spermatozoa will be made elsewhere.

Our particular interest is in genetic effects on the spermatozoan phenotype. There was little immediate background to tell us if such effects are common in Drosophila. We now know of three aspects of genetic behaviour relating to spermatozoan nucleus length within species. Herskowitz and Muller (r954) had reported a strain difference in mean length. In the present work there was strong evidence of genetic interaction affecting both mean length and the degree of skewness in the distribution of lengths. There was also a difference between two parallel strains in their interaction behaviour towards the foundation stock, possibly caused by random drift.

The diploid genotype of the male and the haploid genotype of the developing spermatozoon might each affect the spermatozoan phenotype. There is certainly scope for a "haploid effect" in the high variability in nucleus length within males. However, there was no evidence that differences between $\mathrm{X}$ - and $\mathrm{Y}$-bearing spermatozoa contributed to this variation. Further, the homogeneity of withinmale variances at all levels of analyses suggested that strain-specific factors with haploid expression in spermatozoa were not segregating within males.

\section{SUMMARY}

I. The spermatozoan nucleus of Drosophila melanogaster has been studied with regard to mean length, the within-male variance, and the coefficients of skewness and kurtosis in the within-male distribution. The sources of variation considered were those affecting the progeny classes of a diallel cross and those affecting the subsampling.

2. The material consisted of strains $A$ and $B$ that had already been selected successfully and in parallel for small wing cell size, small body size being a correlated response. Strain $\mathrm{C}$ was the unselected control. No correlated response in spermatozoan nucleus length was detected.

3. In the diallel of $A, B$ and $C$ there was significant genetic interaction affecting length and skewness, but not affecting within-male variance or kurtosis. There were no demonstrable parental strain, maternal or reciprocal effects on length or on the within-male variance.

4. The interaction for mean nucleus length and skewness was specifically between $\mathrm{B}$ and $\mathrm{C}$, the nuclei being longer and more positively skewed in distribution in within-strain progeny than in betweenstrain progeny. (These interactions reappeared in a confirmatory diallel of $B$ and $\mathrm{C}$ only, which showed no parental strain or maternal effects on the within-male variance, or on kurtosis or skewness). No attribute of $\mathrm{A}$ interacted with either B or $\mathrm{C}$. Since $\mathrm{A}$ and $\mathrm{B}$ differ in 
their interaction behaviour towards $\mathrm{C}$, the genes governing nucleus length in $\mathrm{A}$ and $\mathrm{B}$ must be different.

5. In the subsampling, differences in nucleus length within males are thought to be partly biological in origin and partly due to errors in technique and observation. Differences between replicate culture bottles were non-significant for all attributes. Differences in length between males within culture bottles are believed to be due to technique errors. A similar pattern of variation existed for the withinmale variance and kurtosis, but skewness appeared to be homogeneous at all levels of the subsampling.

6 . There was no evidence that $\mathrm{X}$ - and Y-bearing spermatozoa differ in nucleus length, nor of other effects of the haploid gene content on the individual spermatozoon.

7. The basic assumptions underlying the analysis of variance are more closely obeyed if the data are log-transformed, but transformation usually has little effect on the final conclusions.

8. The detailed pattern of variation in spermatozoan nucleus length is highly repeatable from one experiment to another.

9. The results accord with a generalisation that spermatozoan dimensions are controlled genetically but are rarely responsive to environmental factors.

Acknowledgments.-We are grateful to Professor C. H. Waddington for facilities and helpful interest, and to Dr. F. W. Robertson for suggesting the use of the particular strains and for providing the initial stocks. We also thank Dr. A. Robertson and Professor J. L. Jinks for constructive comments on a draft.

\section{REFERENCES}

BEATTY, R. A. rg6ra. Genetics of gametes. V. The frequency distribution of the head length of rabbit spermatozoa, and a search for dimorphism. Proc. Roy. Soc. Edin., B, 68, 72-82.

BEATty, R. A. 1961 b. Genetics of mammalian gametes. Anim. Breeding Abstr., 29, 243-256.

BEATTY, R. A. 1963. The spermatozoa of mouse-strains selected for body weight. Proc. XI internat. Congr. Genetics (Hague, 1963), I, 252-253.

FISHER, R. A. 1930. Statistical Methods for Research Workers. Edinburgh, Oliver and Boyd.

HAYMAN, B. I. 1954. The analysis of variance of diallel tables. Biometrics, 10, 235-244.

HERSKOWITZ, I. H., AND MULLER, H. J. 1954. Evidence against a straight end-to-end alignment of chromosomes in Drosophila spermatozoa. Genetics, 39, 836-850.

RAO, C. R. 1952. Advanced Statistical Methods in Biometric Research. New York, John Wiley.

ROBERTSON, F. W. 196r. The ecological genetics of growth in Drosophila. 4. The influence of larval nutrition on the manifestation of dominance. Genet. Res. (Camb.), 2, 346-36o.

siduU, N. s. 1963. Genetic Effects on the Spermatozoa of Drosophila. Ph.D. Thesis, Edinburgh University.

sidHu, N. s. 1964. A quantitative study of spermatozoan nucleus length in Drosophila melanogaster (Meig.). Proc. Roy. Soc. Edin., B, 68, 327-335.

WEARDEN, s. 1964. Alternative analyses of the diallel cross. Heredity, 19, 669-680. 